\title{
Is Auger-free luminescence present in $\mathrm{CeF}_{3}$ ?
}

\author{
Minoru Itoh ${ }^{\mathrm{a} *}$, Daisuke $\operatorname{Iri}^{\mathrm{a}}$, Mamoru Kitaura $^{\mathrm{b}}$ \\ a Department of Electrical and Electronic Engineering, Shinshu University, \\ Nagano 380-8553, Japan \\ ${ }^{\mathrm{b}}$ Fukui National College of Technology, Sabae 916-8507, Japan
}

\begin{abstract}
It is well known that Auger-free luminescence (AFL) is observable when the condition $E_{\mathrm{g}}>E_{\mathrm{VC}}$ is satisfied, where $E_{\mathrm{g}}$ is the band-gap energy between the lowest unoccupied band and the highest occupied band and $E_{\mathrm{VC}}$ the energy difference between the top of the highest occupied band and the top of the next lower occupied band. From measurements of reflection and x-ray photoelectron spectra, $\mathrm{CeF}_{3}$ is demonstrated to really satisfy this condition. No evidence for AFL is found, nevertheless. The absence of $\mathrm{AFL}$ in $\mathrm{CeF}_{3}$ is related to a characteristic nature of its highest and next lower occupied bands, which are quite different from those of previously studied AFL-materials.
\end{abstract}

PACS Codes: 71.20.Ps, 78.40.Ha, 78.55.Hx, 79.60.-i

Keywords: Auger-free luminescence; cross-luminescence; core-valence luminescence; $\mathrm{Au}-$ ger-free condition; self-trapping; $\mathrm{V}_{\mathrm{k}}$ center; scintillation material; $\mathrm{CeF}_{3}$; $\mathrm{x}$-ray photoelectron spectroscopy; optical reflection; synchrotron radiation

*Corresponding author: Minoru Itoh; Department of Electrical and Electronic Engineering, Faculty of Engineering, Shinshu University, Nagano 380-8553, Japan; Tel: +81-26-269-5261; Fax: +81-26-269-5220; E-mail: itohlab@shinshu-u.ac.jp 


\section{Introduction}

The Auger-free luminescence (AFL) [1], or cross-luminescence [2] and core-valence luminescence [3], is a peculiar type of intrinsic luminescence in wide-gap insulating materials. It arises from interatomic radiative transitions between the highest occupied band and the next lower occupied band, and is observable only when the following condition is satisfied (see Fig. $1)$;

$$
E_{\mathrm{g}}>E_{\mathrm{VC}}
$$

where $E_{\mathrm{g}}$ is the band-gap energy between the lowest unoccupied band and the highest occupied band, and $E_{\mathrm{VC}}$ the energy difference between the top of the highest occupied band and the top of the next lower occupied band. In solid-state physics, the lowest unoccupied, highest occupied, and next lower occupied bands are usually called the conduction, valence, and outermost-core bands, respectively. Under the condition (1), the Auger decay process resulting in electron excitation from the highest occupied band to the lowest unoccupied band is energy-forbidden when a hole is created in the next lower occupied band. The AFL is characterized by a relatively high quantum yield and a high thermal stability, as well as short lifetime of the order of nanoseconds [1-3].

$\mathrm{CeF}_{3}$ crystal is a well-known scintillation material [4], because of the fast $\mathrm{Ce}^{3+} 5 \mathrm{~d} \rightarrow 4 \mathrm{f} \mathrm{lu}$ minescence peaking at 287 and $304 \mathrm{~nm}[5,6]$. In the present study, we measured the reflection and x-ray photoelectron spectra of $\mathrm{CeF}_{3}$, in order to evaluate the values of $E_{\mathrm{g}}$ and $E_{\mathrm{VC}}$. From these results, $\mathrm{CeF}_{3}$ was shown to be certainly an AFL-material. Nevertheless, we could not find any evidence for AFL in this compound. This is the first example in which AFL is not present although the condition (1) is fulfilled.

\section{Experiment}

As-grown crystal of $\mathrm{CeF}_{3}$ was obtained from Prof. A. Yoshikawa of Tohoku University, Sendai. The sample surfaces were mechanically polished for measurements of reflection and emission spectra, and freshly cleaved for x-ray photoelectron spectroscopy (XPS).

For reflection and luminescence measurements, we used synchrotron radiation from the UVSOR ring in the Institute for Molecular Science, Okazaki, as a light source. The incident light was monochromatized with a 1-m Seya-Namioka VUV monochromator. The sample was mounted on the copper holder in a temperature-variable cryostat of He-flow type. Luminescence from the sample surface was dispersed through an Acton SpectraPro-300i monochromator equipped with a liquid-nitrogen cooled charge-coupled device camera (Roper Scientific 100EB-GI). This detection system has a typical spectral resolution of about $5 \mathrm{~nm}$ and a suitable sensitivity in the wavelength range $\lambda_{\mathrm{em}}=200-1000 \mathrm{~nm}$. The excitation wavelength $\lambda_{\mathrm{ex}}$ was changed in 2 or $5 \mathrm{~nm}$ steps, and for each step the emission spectrum was integrated over 5 
seconds. The emission spectra were not corrected for the spectral response of the detection system, while the excitation spectra were corrected for the intensity distribution of the incident light.

XPS spectra were measured at room temperature by using an ESCA spectrometer (ULVAC-PHI 5600), with an excitation source of $\mathrm{Al}$ anode $(K \alpha .1486 .6 \mathrm{eV})$. An electron flood gun was employed to compensate for the sample charging under x-ray irradiation. The overall resolution was about $0.5 \mathrm{eV}$ in the binding-energy region of interest. The base pressure in the sample chamber was less than $6.0 \times 10^{-8} \mathrm{~Pa}$ during the measurements.

\section{Results}

Figure 2 shows the reflection spectrum of $\mathrm{CeF}_{3}$ measured in a wide range up to $35 \mathrm{eV}$ at $6 \mathrm{~K}$. The spectrum in the $4.5-7.5 \mathrm{eV}$ region is expanded in the inset. Three fine peaks are seen at 4.90, 5.25, and $5.60 \mathrm{eV}$, followed by some broad peaks at around 5.90, 6.25, and $6.80 \mathrm{eV}$. The spectrum of Fig. 2 is in fairly good agreement with the earlier result of $\mathrm{CeF}_{3}$ by Olson, Piacentini, and Lynch [7], who measured the reflection spectrum of single crystal in the 10-34 eV region and the absorption spectrum of thin film in the $4-9 \mathrm{eV}$ region. The peaks appearing in the inset of Fig. 2 are apparently due to the $4 \mathrm{f} \rightarrow 5 \mathrm{~d}$ transitions of $\mathrm{Ce}^{3+}$ ions. The reflection spectrum around the lowest-energy transition shows a dispersive shape, from which the resonance energy is estimated to be $5.0 \mathrm{eV}$. This value coincides well with the lowest absorption energy of thin films in ref [7]. An intense band at $11.0 \mathrm{eV}$ with shoulder-like structure around $13.5 \mathrm{eV}$ is attributed to the transitions from the F $2 p$ state to the Ce $5 \mathrm{~d}$, 6s states. Three or four broad structures are observed in the $20-30 \mathrm{eV}$ region, which are probably attributed to the $5 \mathrm{p} \rightarrow 5 \mathrm{~d}$ and 6 s transitions of $\mathrm{Ce}^{3+}[7]$.

The XPS spectrum is shown in Fig. 3, where the binding energy is given relative to the top of the highest occupied band. The overall structures are similar to those measured by Park and Oh [8], although the resolution of our spectrum is better than their one. The highest occupied band originates from the $\mathrm{Ce} 4 \mathrm{f}$ state. The next lower occupied band peaking at around $6 \mathrm{eV}$ is attributed to the F $2 p$ state. A striking feature of Fig. 3 is the existence of a gap of $2.4 \pm 0.5 \mathrm{eV}$ between the $\mathrm{Ce} 4 \mathrm{f}$ band and the $\mathrm{F} 2 \mathrm{p}$ band. The doublet structure around $18 \mathrm{eV}$ is ascribed to the spin-orbit split Ce 5p state. Two peaks at 27 and $35 \mathrm{eV}$ are assigned to the $\mathrm{F} 2 \mathrm{~s}$ and $\mathrm{Ce} 5 \mathrm{~s}$ states, respectively.

The contour plot of the emission-excitation spectrum at $6 \mathrm{~K}$ is presented in the $\left(\lambda_{\mathrm{em}}, \lambda_{\mathrm{ex}}\right)$ plane of Fig. 4. The $\mathrm{Ce}^{3+} 5 \mathrm{~d} \rightarrow 4 \mathrm{f}$ luminescence is clearly observed, having two peaks at $\lambda_{\mathrm{em}}=$ 287 and $304 \mathrm{~nm}(4.32$ and $4.08 \mathrm{eV})$. This luminescence is efficiently excited with photons in the region of $\lambda_{\mathrm{ex}}=260-178 \mathrm{~nm}(4.8-7.0 \mathrm{eV})$, which corresponds to the $4 \mathrm{f} \rightarrow 5 \mathrm{~d}$ absorption bands of $\mathrm{Ce}^{3+}$ ions [7]. It is also stimulated with high-energy photons at $\lambda_{\mathrm{ex}}<170 \mathrm{~nm}(>7.3 \mathrm{eV})$. A 
weak long-wavelength emission band of extrinsic origin appears under the excitation at $\lambda_{\mathrm{ex}}>$ $260 \mathrm{~nm}(<4.8 \mathrm{eV})$. No other luminescence was observed at $6 \mathrm{~K}$ and also at $300 \mathrm{~K}$, even though the excitation energy was extended up to $25 \mathrm{eV}\left(\lambda_{\mathrm{ex}}=50 \mathrm{~nm}\right)$.

\section{Discussion}

From the present results of reflection and XPS spectra, we can obtain the values of $E_{\mathrm{g}}$ and $E_{\mathrm{VC}}$, as well as the highest occupied-band width $\Delta E_{\mathrm{V}}$ and the next lower occupied-band width $\Delta E_{\mathrm{C}}$. The value of $E_{\mathrm{g}}$ is determined to be $5.0 \pm 0.1 \mathrm{eV}$ from the lowest dispersive reflection in the inset of Fig. 2. The XPS spectrum of Fig. 3 gives $E_{\mathrm{VC}}=3.9 \pm 0.5 \mathrm{eV}, \Delta E_{\mathrm{V}}=1.5 \pm 0.5 \mathrm{eV}$, and $\Delta E_{\mathrm{C}}=4.3 \pm 0.5 \mathrm{eV}$. The main source of uncertainty in the obtained values comes from the insufficient resolution $(0.5 \mathrm{eV})$ of XPS spectra. The energy-level diagram of $\mathrm{CeF}_{3}$ determined from the present experiments is presented in Fig. 5. From this figure, it can be said that the $\mathrm{Au}-$ ger-free condition (1) is fulfilled in $\mathrm{CeF}_{3}$, even if the experimental error of each parameter is most severely evaluated. We can thus expect the appearance of AFL for this material.

Direct evidence for AFL comes from the measurement of excitation spectra. From Fig. 1, it is expected that AFL has the excitation threshold at $\lambda_{\mathrm{ex}}=E_{\mathrm{g}}+E_{\mathrm{VC}} \approx 140 \mathrm{~nm}(8.9 \mathrm{eV})$. Furthermore, the photons of AFL will be emitted in the $\lambda_{\mathrm{em}}$ region between $E_{\mathrm{VC}}(\approx 320 \mathrm{~nm})$ and $E_{\mathrm{VC}}-$ $\Delta E_{\mathrm{V}}(\approx 520 \mathrm{~nm})$, if it is assumed that the lattice relaxation induced by a hole created in the next lower occupied band is neglected (rigid lattice model). In Fig. 4, there is no luminescence signal in the $\left(\lambda_{\mathrm{em}}, \lambda_{\mathrm{ex}}\right)$ area expected for AFL; $320 \mathrm{~nm}<\lambda_{\mathrm{em}}<520 \mathrm{~nm}$ and $\lambda_{\mathrm{ex}}<140 \mathrm{~nm}$. That is to say, $\mathrm{AFL}$ is absent in $\mathrm{CeF}_{3}$, although the condition (1) is satisfied.

In general, electrons interact strongly with phonons in insulating materials, especially in metal halides. However, there is a significant difference for holes, depending on whether the occupied band is built up by the ground state of halogen ions or metal ions. As is well established in alkali halides [9], when a hole is generated in the highest occupied band made from halogen states, it becomes self-trapped by forming an $\mathrm{X}_{2}^{-}$molecular ion ( $\mathrm{X}$ denotes halogen atom) during its lifetime. The trap depth $\Delta E$, i.e., lattice relaxation energy around a hole, has been estimated by Inouye and Pong [10], by leaving the relaxation of $\mathrm{X}_{2}^{-}$-type out of consideration. They have obtained $\Delta E=1.9 \mathrm{eV}$ for $\mathrm{RbF}$ as an example. If the lattice relaxation of $\mathrm{X}_{2}^{-}$-type is further taken into account, the value of $\Delta E$ is expected to get a remarkable increase. On the other hand, it has been pointed out [11] that when a hole is generated in the next lower occupied band made from metal states, it is also self-trapped, not deeply but very shallowly, on an isolated metal ion.

In AFL-materials ever found [1-3], the highest occupied (valence) band is mainly formed by the ground state of halogen ions, while the next lower occupied (outermost-core) band is built up by the ground state of metal ions. Interestingly, this situation is in reverse in $\mathrm{CeF}_{3}$, in which the highest occupied band is built up by the $4 \mathrm{f}$ state of $\mathrm{Ce}^{3+}$ ions and the next lower occupied 
band has highly $2 \mathrm{p}$ character of $\mathrm{F}^{-}$ions. In this connection, it is important to notice that the $\mathrm{CeF}_{3}$ crystals are bound by the next lower occupied F 2 p electrons, not by the highest occupied Ce $4 \mathrm{f}$ electrons. Therefore, one may rather say that the F $2 p$ state forms the valence band [12], and the $\mathrm{Ce} 4 \mathrm{f}$ state forms the localized band situating above the valence band. The physical essence of this paper is not at all influenced by such terminological difference.

The previously studied AFL is usually attributed to the radiative recombination of free valence electrons with localized or nearly-free core holes [1-3]. However, this is not an essential character of AFL. For example, in mixed $\mathrm{CsF}_{1-x} \mathrm{Cl}_{x}$ system, the localized $\mathrm{Cl} 3 \mathrm{p}$ state (impurity band) is situated $1-2 \mathrm{eV}$ above the top of the $\mathrm{F} 2 \mathrm{p}$ valence band, in which the impurity-associated AFL appears as a result of the radiative transition of localized electrons on $\mathrm{Cl}^{-}$ ions to $\mathrm{Cs}^{+} 5 \mathrm{p}$ core holes, in addition to the host AFL [13]. In the present study, we are concerned with the Auger-free radiative recombination of localized electrons with free holes in undoped material.

We must now ask why the AFL is absent in $\mathrm{CeF}_{3}$. It is essential for the production of AFL to generate a hole in the next lower occupied band. Until now, we have always dealt with the holes generated on metal ions in AFL-materials. Shallow self-trapping of such holes results in the low-energy tail of AFL [11], but the main part of AFL appears in the vicinity of the $\lambda_{\mathrm{em}}$ region predicted from the rigid lattice model. On the other hand, in $\mathrm{CeF}_{3}$, the hole is generated on an $\mathrm{F}^{-}$ ion. This hole is supposed to form an $\mathrm{F}_{2}^{-}$molecular ion, as in the case of valence holes in alkali halides. Such a self-trapped hole could introduce large relaxation energy enough to overcome the gap of $2.4 \pm 0.5 \mathrm{eV}$, thus merging nonradiatively into the highest occupied band composed of the $\mathrm{Ce} 4 \mathrm{f}$ state. The argument mentioned above is a plausible explanation for the fact that the AFL is not present in $\mathrm{CeF}_{3}$ that holds the condition (1), and is supported by the EPR experiment in which no $\mathrm{V}_{\mathrm{k}}$ centers $\left(\mathrm{F}_{2}^{-}\right.$molecular ions) are found for $\mathrm{CeF}_{3}$ [14].

Among rare-earth trifluorides, $\mathrm{LaF}_{3}$, lacking $4 \mathrm{f}$ electrons, has many studies on its optical

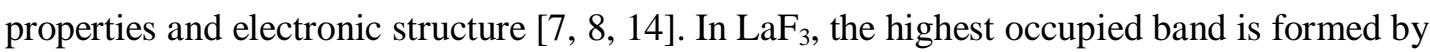
the F $2 p$ state and the next lower occupied band is formed by the La 5p state. The situation is the same as that in AFL-materials studied before. We can estimate $E_{\mathrm{g}} \approx 10 \mathrm{eV}$ from the reflection spectrum [7] and $E_{\mathrm{VC}} \approx 10 \mathrm{eV}$ from the XPS spectrum [8]. Therefore, the observation of AFL is not probably expected for $\mathrm{LaF}_{3}$. In $\mathrm{PrF}_{3}, \mathrm{NdF}_{3}$, and $\mathrm{DyF}_{3}$, the rare-earth $4 \mathrm{f}$ states are isolated above the $\mathrm{F} 2 \mathrm{p}$ band [7], similarly to the case of $\mathrm{CeF}_{3}$. Unfortunately, we can discuss nothing on the observability of AFL in these materials, because the XPS data are not available in the literature.

Finally, we make a brief comment on $\mathrm{CeF}_{3}$. If the self-trap depth of the holes generated on $\mathrm{F}^{-}$ ions in $\mathrm{CeF}_{3}$ is no so large, the $\mathrm{AFL}$ will be observed in the $\lambda_{\mathrm{em}}$ region longer than $E_{\mathrm{VC}}-\Delta E_{\mathrm{V}}(\approx$ $520 \mathrm{~nm}$ ). In the present experiment, we examined the emission spectra in the wavelength range 
up to $1000 \mathrm{~nm}$, without successful observation of luminescence signal related to AFL. Further studies using infrared detection system are required to check this possibility.

\section{Acknowledgements}

The authors gratefully thank Prof. A. Yoshikawa for providing the single crystal of $\mathrm{CeF}_{3}$, and Mr. T. Aoki, Mr. H. Mitani, and Mr. K. Sakaguchi for their assistance in the course of this study. The present work was partially supported by the Joint Studies Program of the Institute for Molecular Science, Okazaki.

\section{References}

[1] M. Itoh, "Recent Research Developments in Physics, Vol. 4" (Transworld Research Network, Trivandrum, 2003), Chap. 11.

[2] V.N. Makhov, "NATO Security through Science Series B: Physics and Biophysics" (Springer, 2007), p.349.

[3] P.A. Rodnyi, Radiat. Meas. 38 (2004) 343.

[4] E. Auffray, et al., Nucl. Instrum. Methods Phys. Res. A 378 (1996) 171.

[5] D.F. Anderson, Nucl. Instrum. Methods Phys. Res. A 287 (1990) 606.

[6] C.W.E. van Eijk, Nucl. Instrum. Methods Phys. Res. A 460 (2001) 1.

[7] C.G. Olson, M. Piacentini, D.W. Lynch, Phys. Rev. B 18 (1978) 5740.

[8] K.-H. Park, S.-J. Oh, Phys. Rev. B 48 (1993) 14833.

[9] Y. Toyozawa, "Optical Processes in Solids" (Cambridge University Press, Cambridge, 2003), Chap. 9.

[10] C.S. Inouye, W. Pong, Phys. Rev. B 15 (1977) 2265.

[11] M. Fukaya, Y. Kayanuma, M. Itoh, J. Phys. Soc. Jpn. 71 (2002) 2557.

[12] The assignment of the F $2 \mathrm{p}$ state to the valence band leads to the band-gap energy $=5.0 \mathrm{eV}$ $+3.9 \mathrm{eV} \approx 8.9 \mathrm{eV}$ from the present experiments. This is in satisfactorily good agreement with the previously calculated value $(\approx 10 \mathrm{eV})$; H. Merenga, J. Andriessen, C.W.E. van Eijk, Radiat. Meas. 24 (1995) 343.

[13] M. Itoh, N. Ohno, H. Yoshida, S. Hashimoto, K. Kan'no, M. Kamada, J. Electron Spectrosc. Relat. Phenom. 79 (1996) 117.

[14] E.D. Thoma, H. Shields, Y. Zhang, B.C. McCollum, R.T. Williams, J. Lumin. 71 (1997) 93. 


\section{Figure Captions}

Fig. 1. Schematic energy-level diagram of insulating materials satisfying the Auger-free condition $E_{\mathrm{g}}>E_{\mathrm{VC}} . E_{\mathrm{g}}$ : the band-gap energy between the lowest unoccupied band and the highest occupied band, $E_{\mathrm{VC}}$ : the energy difference between the top of the highest occupied band and the top of the next lower occupied band, $\Delta E_{\mathrm{V}}$ : the highest occupied-band width, and $\Delta E_{\mathrm{C}}$ : the next lower occupied-band width.

Fig. 2. Reflection spectrum of $\mathrm{CeF}_{3}$ in a wide range up to $35 \mathrm{eV}$ at $6 \mathrm{~K}$. The inset shows the spectrum in the $4.5-7.5 \mathrm{eV}$ region in an expanded scale.

Fig. 3. XPS spectrum of $\mathrm{CeF}_{3}$. The binding energy is given relative to the top of the highest occupied band.

Fig. 4. The luminescence intensity at $6 \mathrm{~K}$ is plotted as functions of excitation and emission wavelengths.

Fig. 5. Energy-level diagram of $\mathrm{CeF}_{3}$ determined from the present experiments. 


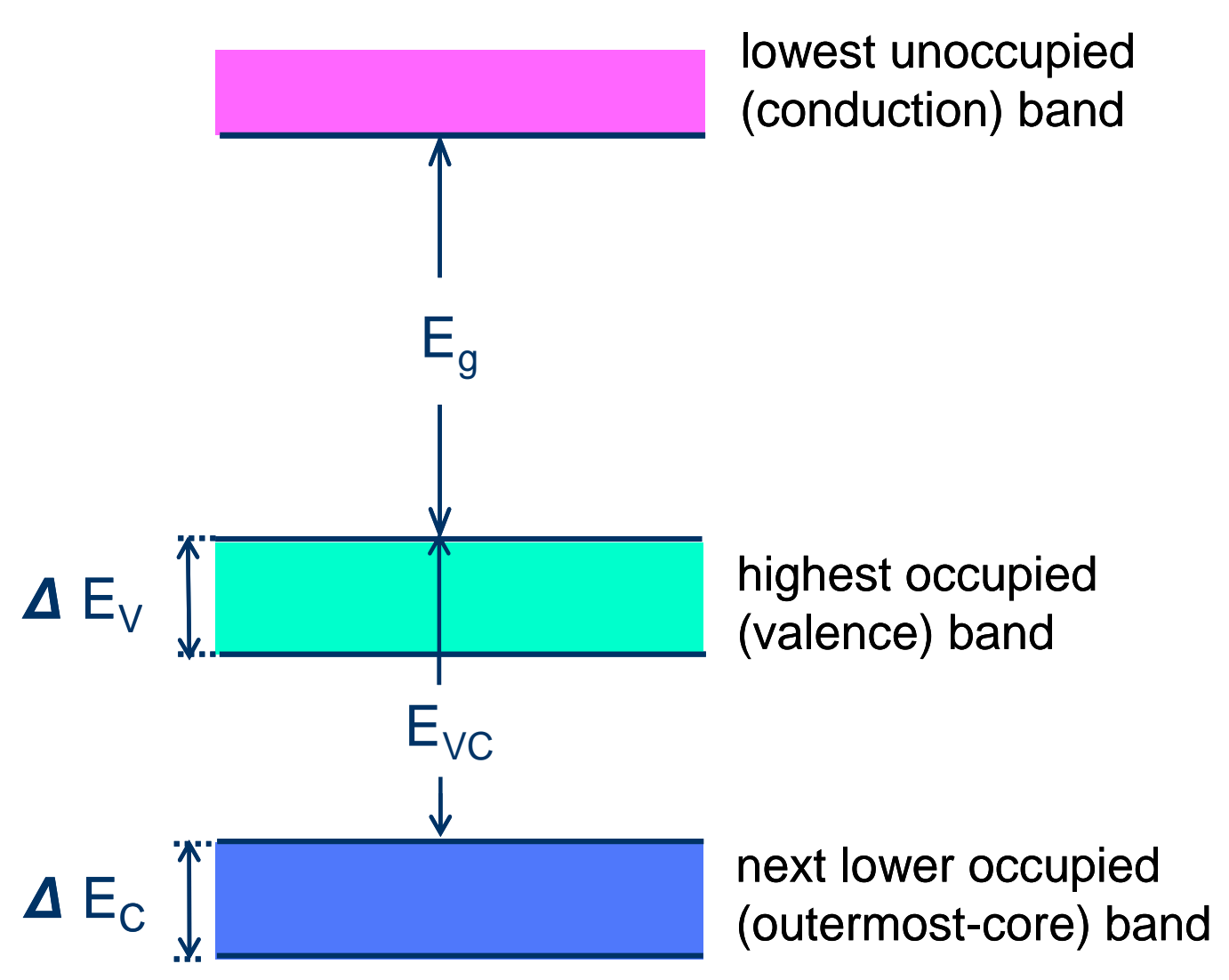

Fig. 1 


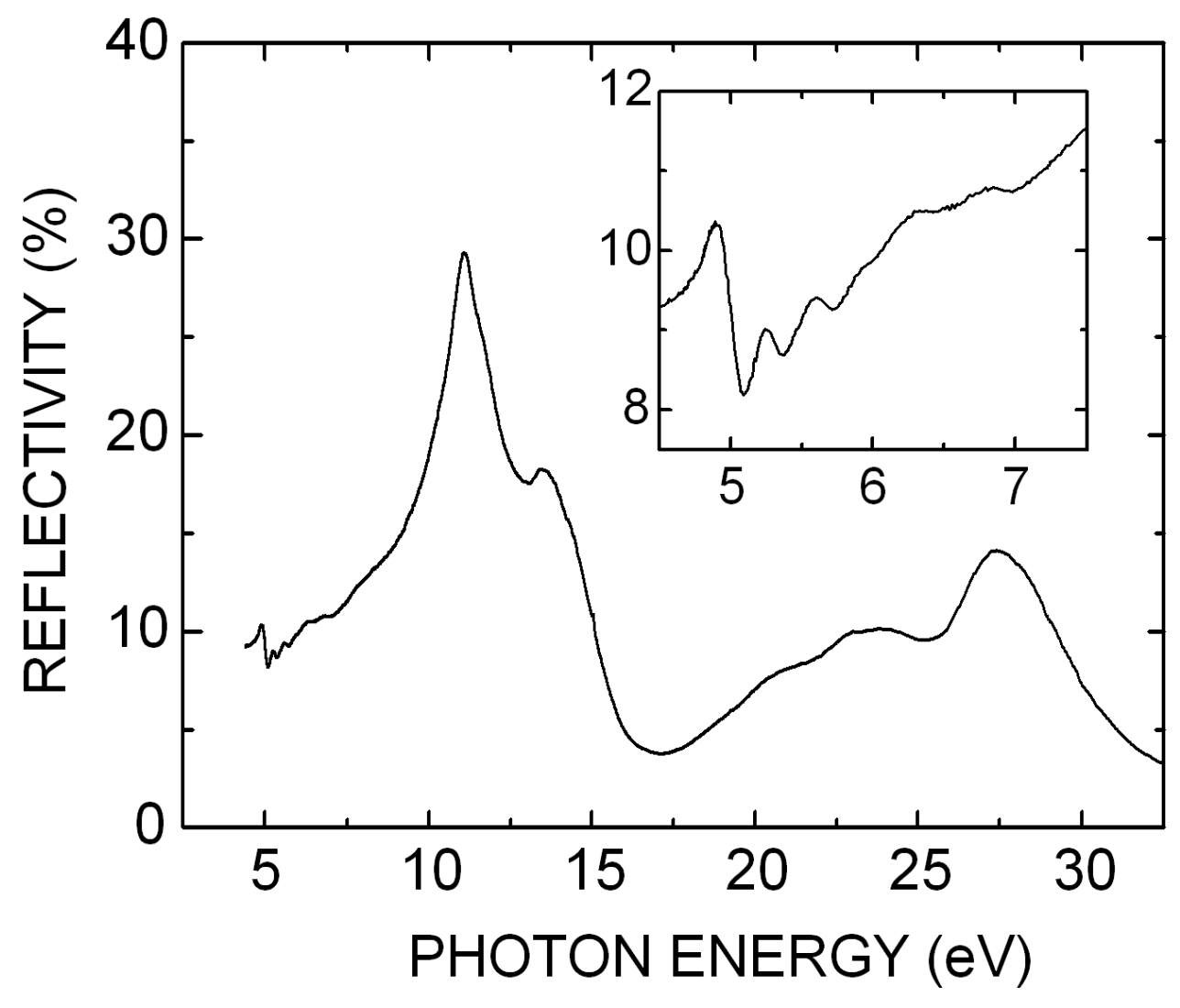

Fig. 2 


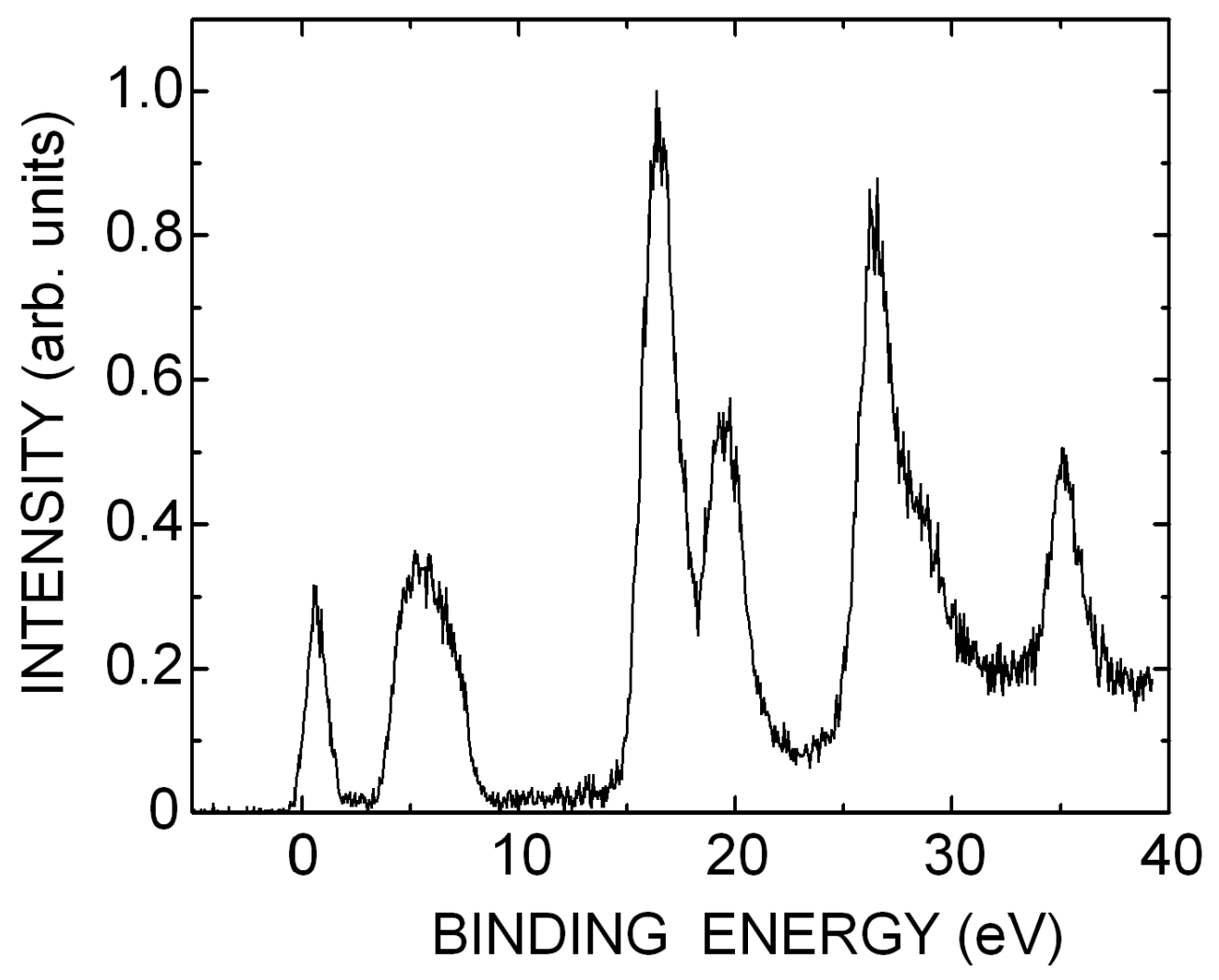

Fig. 3 


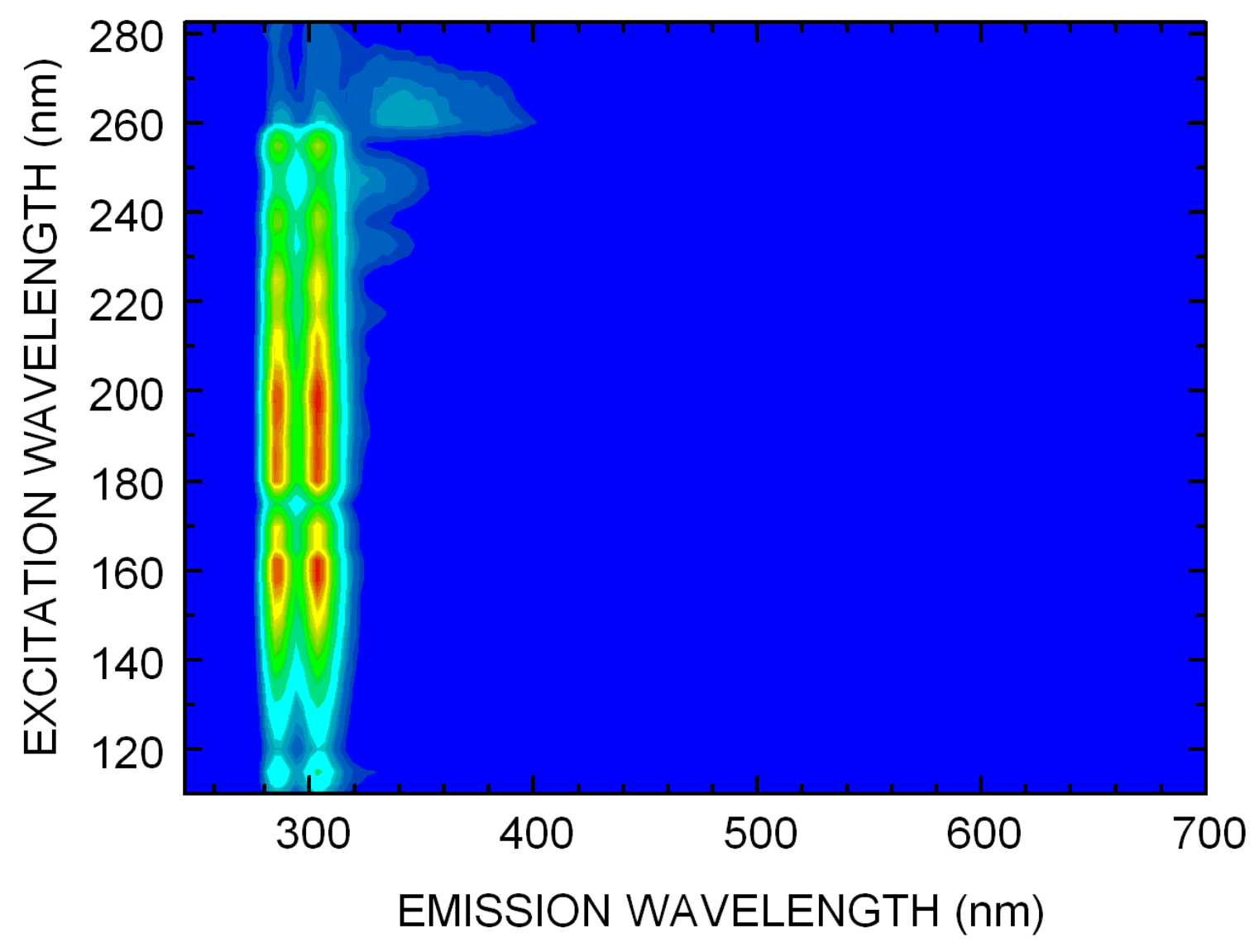

Fig. 4 


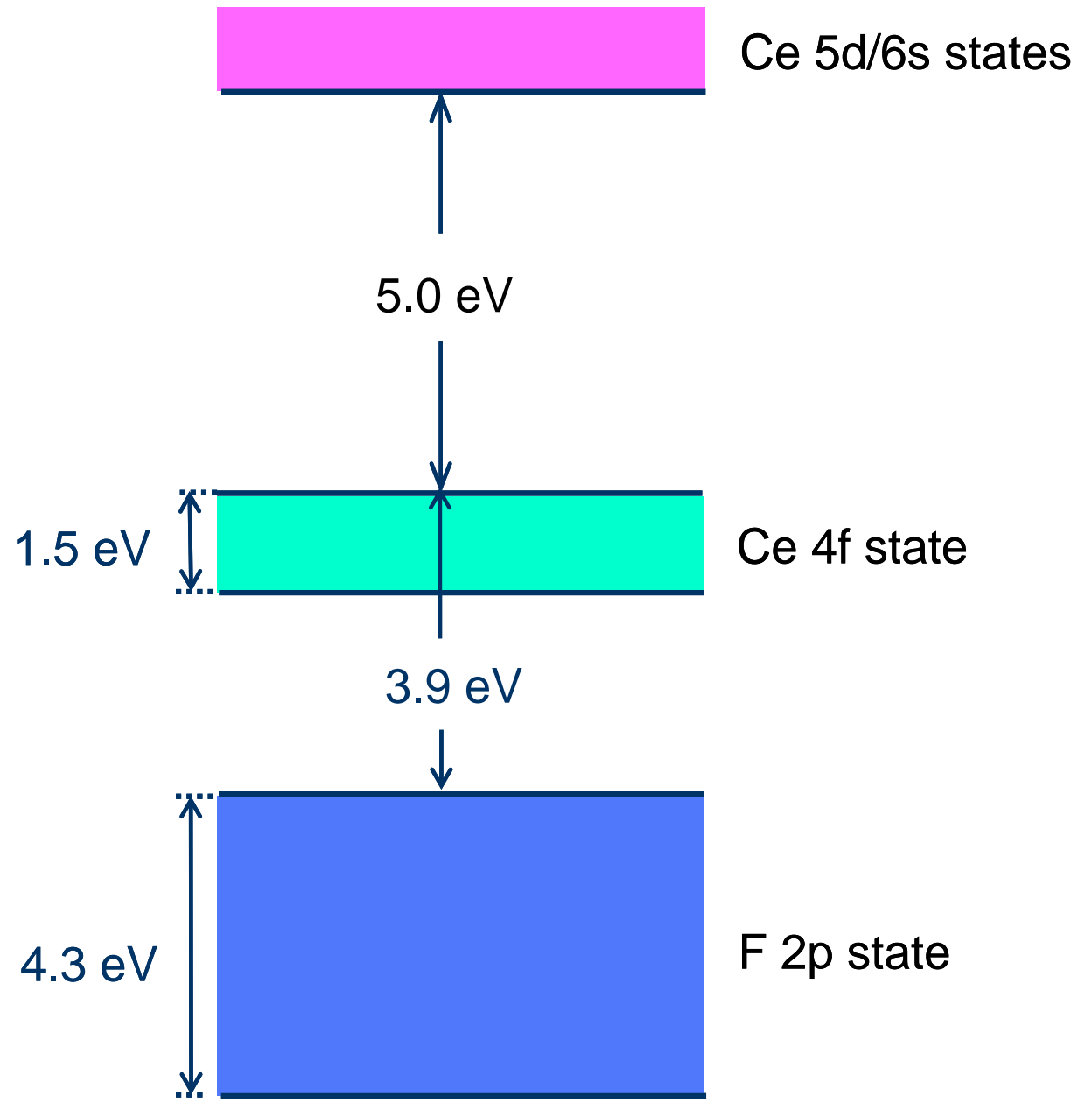

Fig. 5 